\title{
Grand Equilibrium: vapour-liquid equilibria by a new molecular simulation method
}

\author{
Jadran Vrabed*, Hans Hasse \\ Institut für Technische Thermodynamik und Thermische Verfahrenstechnik, \\ Universität Stuttgart, D-70550 Stuttgart, Germany
}

Keywords: molecular simulation, vapour-liquid equilibria, Gibbs ensemble Monte Carlo, NpT plus test particle method, Lennard-Jones, Grand canonical ensemble

Number of pages: 20
Number of tables: 7
Number of figures: 5
Running title: VLE by Grand Equilibrium

Running title: VLE by Grand Equilibrium

\footnotetext{
* author for correspondence, tel.: +49-711/685-6107, fax: +49-711/685-7657, email: vrabec@itt.uni-stuttgart.de
} 


\begin{abstract}
A new molecular simulation method for the calculation of vapour-liquid equilibria of mixtures is presented. In this method, the independent thermodynamic variables are temperature and liquid composition. In the first step, one isobaric isothermal simulation for the liquid phase is performed, in which the chemical potentials of all components and their derivatives with respect to the pressure, i.e., the partial molar volumes, are calculated. From these results, first order Taylor series expansions for the chemical potentials as functions of the pressure $\mu_{i}(p)$ at constant liquid composition are determined. That information is needed, as the specified pressure in the liquid will generally not be equal to the equilibrium pressure, which has to be found in the course of a vapour simulation. In the second step, one pseudo grand canonical simulation for the vapour phase is performed, where the chemical potentials are set according to the instantaneous pressure $p^{v}$ using the previously determined function $\mu_{i}\left(p^{v}\right)$. In this way, results for the vapour pressure and vapour composition are achieved which are consistent to the given temperature and liquid composition. The new method is applied to the pure Lennard-Jones fluid, a binary, and a ternary mixture of Lennard-Jones spheres and shows very good agreement with corresponding data from the literature.
\end{abstract}




\section{Introduction}

Due to the rapid increase of available computing power, molecular simulation is developing to become a standard tool in chemical engineering and related areas. It can provide a better understanding of industrial processes and the underlying physics on the molecular level. Furthermore, molecular simulation has the capability to predict phase equilibria more reliably than classical thermodynamic models. The calculation of vapour-liquid equilibria by molecular simulation is a longstanding and important task. In the last 15 years a variety of methods for this purpose have been presented.

There are, among others, thermodynamic scaling [1,2], histogram reweighting [3]4, GibbsDuhem integration [5,6], NpT plus test particle method [10]1], various extensions of it to other ensembles [7,8,9], and, most widely used, the Gibbs Ensemble Monte Carlo method (GEMC) [12,13]. A discussion of the different approaches can be found in the reviews [14,15].

The GEMC specifies the thermodynamic variables temperature, global composition, and either global volume or pressure. Both phases are simulated simultaneously in separate volumes, between which real particles are exchanged in order to ensure phase equilibrium. On the one hand, this set of specified thermodynamic variables is in many cases not convenient, i.e., when bubble points or dew points are needed, where temperature or pressure and the composition of one phase is specified. On the other hand, the simultaneous simulation of both phases has the disadvantage that fluctuations occuring in one phase influence the other phase directly. This can result in unfavourable dynamic behaviour of the simulation, e.g., close to the critical point fluctuations can lead to phase reversal in the two volumes. Furthermore, real particle exchange between the two phases is a major cause of statistical uncertainties. If the liquid is dense and/or consists of strongly inter- 
acting particles, the insertion and deletion of real particles is awkward and perturbs the liquid causing high statistical uncertainties.

In this work, the Grand Equilibrium method for the calculation of vapour-liquid equilibria of multi-component mixtures is presented. This method circumvents the above mentioned problems. The specified thermodynamic variables are temperature and liquid composition, two independent simulations for both phases are performed and no exchange of real particles in the liquid phase needs to be done.

The Grand Equilibrium method has some common features with the NpT plus test particle method for mixtures [11]6]. In fact, the treatment of the liquid phase is identical. The difference lies in the procedure for the vapour phase, where only one simulation has to be performed and no prior knowledge of the vapour-liquid equilibrium is neccessary. Furthermore, the present method is related to the method of Ungerer et al. [17,18], but avoids introducing direct coupling between the phases. Similar to the work of Esobedo [19,20], it employs pseudo ensemble simulations.

The Grand Equilibrium method is tested in the present work on the pure Lennard-Jones fluid, a binary, and a ternary Lennard-Jones mixture and the results are compared to literature data obtained by other methods. 


\section{Method}

The basic idea of the Grand Equilibrium method is to evaluate the chemical potentials in the liquid as functions of the pressure at a given temperature and liquid composition and to use these functions to set the chemical potentials in a pseudo grand canonical ensemble simulation for the vapour phase. The phase equilibrium conditions are directly introduced in the vapour simulation, which yields vapour pressure, vapour composition and other properties on the dew line.

Let us consider a mixture consisting of an arbitrary number of components $i=\mathrm{A}, \mathrm{B}, \mathrm{C}, \ldots$ and a given set of intermolecular potentials between all species. Having set the independent thermodynamic variables temperature $T$ and liquid composition $\mathbf{x}=x_{A}, x_{B}, x_{C}, \ldots$, one simulation in the isobaric isothermal (NpT) ensemble is carried out at an arbitrarily chosen pressure $p^{l}$, preferrably not too far from the vapour pressure $p_{\sigma}$. This simulation yields the density $\rho^{l}=\rho\left(T, \mathbf{x}, p^{l}\right)$ and the enthalpy $h^{l}=h\left(T, \mathbf{x}, p^{l}\right)$. Applying fluctuation formulae in the NpT ensemble allows the derivatives of the density and enthalpy with respect to the pressure at constant temperature and constant composition to be obtained from that simulation. The derivative of the density is calculated from [21]

$$
\left(\frac{\partial \rho}{\partial p}\right)_{T, \mathbf{x}}=\rho \beta_{T}=\frac{1}{k T} \cdot \frac{N}{<V>^{2}} \cdot\left(<V^{2}>-<V>^{2}\right),
$$

where $V$ denotes the instantaneous volume, $N$ the total number of particles, $k$ the Boltzmann constant, $\beta_{T}$ the isothermal compressibility, and the brackets $<>$ indicate averaging in the NpT ensemble. The derivative of the enthalpy is calculated from [21]

$$
\left(\frac{\partial h}{\partial p}\right)_{T, \mathbf{x}}=\frac{1}{N} \cdot\left[\frac{1}{k T} \cdot(<H><V>-<H V>)+<V>\right]
$$

where $H$ denotes the instantaneous enthalpy. 
For the present method it is essential to additionally calculate the chemical potentials $\mu_{i}$ and the partial molar volumes $v_{i}$ of all components from this liquid phase simulation. This can be done, e.g., by Widom's test particle insertion [22], according to which

$$
\mu_{i}=\mu_{i}^{\mathrm{id}}(T)+k T \cdot \ln x_{i}-k T \cdot \ln <V \cdot \exp \left(-\psi_{i} / k T\right)>/ N
$$

where $\psi_{i}$ denotes the potential energy of a test particle of species $i$ and $\mu_{i}^{i d}(T)$ the part of the ideal chemical potential which only depends on the temperature. It is convenient to define the residual chemical potential of component $i$ as

$$
\tilde{\mu}_{i}=\left[\mu_{i}-\mu_{i}^{\mathrm{id}}(T)\right] / k T \text {. }
$$

Widom's test particle insertion also yields the partial molar volumes [23]

$$
v_{i}=\frac{<V^{2} \cdot \exp \left(-\psi_{i} / k T\right)>}{<V \cdot \exp \left(-\psi_{i} / k T\right)>}-<V>
$$

It is important to note that the Grand Equilibrium method is not tied to Widom's test particle insertion, which is known to yield high statistical uncertainties in very dense and/or strongly interacting fluids. In such cases, other more sophisticated methods to determine the chemical potentials, such as gradual insertion in extended ensembles [24,25,26], can be applied alternatively.

The complete set of thermodynamic quantities that have to be calculated from one NpT simulation at given $T, \mathbf{x}, p^{l}$ in the liquid phase is $\rho^{l}, h^{l}, \beta_{T}^{l},(\partial h / \partial p)_{T, \mathbf{x}}^{l}, \mu_{i}^{l}$, and $v_{i}^{l}$. With these quantities, the desired chemical potentials, as functions of the pressure, are obtained from first order Taylor series expansions [11]

$$
\tilde{\mu}_{i}(p) \approx \tilde{\mu}_{i}^{l}+v_{i}^{l} / k T \cdot\left(p-p^{l}\right)
$$


with

$$
\left(\frac{\partial \mu_{i}^{l}}{\partial p}\right)_{T, \mathbf{x}}=v_{i}^{l} .
$$

It should be mentioned that in typical applications, the accuracy of equation (6) is not influenced significantly by neglecting the higher order terms, but rather by the accuracy of $\mu_{i}^{l}$ and $v_{i}^{l}$ achieved in the simulation. Furthermore, in a liquid phase not too close to the critical point the chemical potentials are only weak functions of the pressure. 1

Having determined the $\mu_{i}(p)$ from the Taylor series expansions, according to equation (6) by one $\mathrm{NpT}$ simulation in the liquid mixture, one vapour simulation in a pseudo grand canonical ensemble (pseudo- $\mu \mathrm{VT}$ ) has to be carried out. In a common grand canonical ensemble $(\mu \mathrm{VT})$ simulation [27,28,29], the temperature, the volume, and the chemical potentials of all components are set as fixed values. In the present pseudo- $\mu \mathrm{VT}$ simulation the temperature and the volume are set in the common way. But instead of setting fixed values for the chemical potentials, they are set as functions of the instantaneous pressure in the vapour $p^{v}$ using equation (6) to find $\mu_{i}\left(p^{v}\right)$. Applying this procedure, it is ensured that equilibrium between the liquid phase and vapour phase is imposed.

During a common $\mu \mathrm{VT}$ simulation the chemical potentials are set through insertion and deletion of particles by the comparison between the resulting potential energy change and the desired fixed chemical potential. In the proposed pseudo- $\mu \mathrm{VT}$ ensemble, in which the values of the chemical potential are found from the function $\mu_{i}\left(p^{v}\right)$, the acceptance probability $P_{a c c}$ for particle insertion of species $i$ writes as

$$
P_{a c c}(N \rightarrow N+1)=\min \left(1, \frac{V}{N_{i}+1} \cdot \exp \left\{\tilde{\mu}_{i}\left(p^{v}\right)-[U(N+1)-U(N)] / k T\right\}\right)
$$

$\overline{1 \text { If equation (6) }}$ is needed in the near-critical region and no good guess for $p^{l}$ is available, succesive simulations in the liquid phase can be necessary. 
where $N_{i}$ denotes the number of particles of species $i$ and $U(N+1)$ the configurational energy of the system with $N+1$ particles. The acceptance probability for particle deletion of species $i$ writes as

$$
P_{a c c}(N \rightarrow N-1)=\min \left(1, \frac{N_{i}}{V} \cdot \exp \left\{-\tilde{\mu}_{i}\left(p^{v}\right)+[U(N)-U(N-1)] / k T\right\}\right)
$$

Starting from an arbitrarily chosen low density state point, the vapour simulation is forced by this procedure to change its state to the corresponding phase equilibrium state point on the dew line. The vapour pressure $p_{\sigma}$ and the vapour composition on the dew line $\mathbf{y}=y_{A}, y_{B}, y_{C}, \ldots$ are then simple ensemble averages in the vapour simulation in the pseudo- $\mu \mathrm{VT}$ ensemble. The same holds for other properties on the dew line, such as dew density and dew enthalpy.

Experience shows that the pseudo- $\mu \mathrm{VT}$ simulation moves rapidly into the vicinity of the phase equilibrium state point. This process happens in the equilibration period. During the production period the pressure just fluctuates around its constant average. These inherent fluctuations in the pressure impose fluctuations on the set chemical potentials through equation (6) . Such an interdependence characterizes pseudo statistical ensembles. Figure 1 gives an impression of a typical equilibration process obtained using the procedure described above for a binary Lennard-Jones mixture. After an initial equilibration of 1000 Monte Carlo loops in the canonical ensemble (NVT), a second equilibration in the pseudo$\mu \mathrm{VT}$ ensemble is started. It can be observed that the instantaneous values of pressure, density, and vapour composition reach the vicinity of equilibrium after only 1000 Monte Carlo loops. Due to the experience with various mixtures including nonspherical and polar fluids [31], it can be stated that this process is very robust and independent on the initial density and composition in the vapour simulation.

With the knowledge of the vapour pressure, other thermodynamic properties of interest 
on the bubble line can be calculated easily, e.g., the bubble density

$$
\rho^{\prime} \approx \rho^{l}+\rho^{l} \beta_{T}^{l} \cdot\left(p_{\sigma}-p^{l}\right)
$$

the bubble enthalpy

$$
h^{\prime} \approx h^{l}+\left(\frac{\partial h^{l}}{\partial p}\right)_{T, \mathbf{x}} \cdot\left(p_{\sigma}-p^{l}\right)
$$

and the chemical potentials in phase equilibrium

$$
\tilde{\mu}_{i \sigma} \approx \tilde{\mu}_{i}^{l}+v_{i}^{l} / k T \cdot\left(p_{\sigma}-p^{l}\right)
$$

The present method can be applied for the calculation of dew points as well. Therefore the phases simply have to be inverted, i.e. one NpT simulation in the vapour phase and one pseudo- $\mu V T$ simulation in the liquid phase have to be performed. In this case it faces the awkward insertion and deletion of real particles in the liquid phase, but still has the advantage of independent simulations runs.

Furthermore, the Grand Equilibrium method can be applied to the more challenging liquid-liquid equilibria as well. First results [30] confirm the applicability and demonstrate high accuracy. 


\section{Applications}

In order to test the Grand Equilibrium method, it has been applied to the pure LennardJones fluid, a binary, and a ternary Lennard-Jones model mixture. The Lennard-Jones intermolecular potential acting between two particles of species $i$ and $j$ is defined by

$$
u_{i j}(r)=4 \varepsilon_{i j}\left[\left(\frac{\sigma_{i j}}{r}\right)^{12}-\left(\frac{\sigma_{i j}}{r}\right)^{6}\right],
$$

where $r$ is the intermolecular distance and $\sigma_{i j}$ and $\varepsilon_{i j}$ are the specific size and energy parameters. In the case of a Lennard-Jones mixture, the full set of size and energy parameters for all interactions has to be specified. Unlike interaction parameters are often expressed on the basis of the like interaction parameters by

$$
\sigma_{i j}=\left(\sigma_{i}+\sigma_{j}\right) / 2
$$

and

$$
\varepsilon_{i j}=\xi_{i j} \cdot \sqrt{\varepsilon_{i} \cdot \varepsilon_{j}}
$$

where $\xi_{i j}$ is a binary interaction parameter.

\subsection{Pure Lennard-Jones fluid}

The pure Lennard-Jones fluid, being a very simple but realistic molecular model, has been studied extensively by many authors. Its vapour-liquid equilibria are known with high accuracy $32,33,34]$, so that it is a favourable test fluid here.

In a pure fluid the size and energy parameters are constant for all interactions, so that

$\sigma_{i j}=\sigma$ and $\varepsilon_{i j}=\varepsilon$. All thermodynamic quantities are reduced in the usual way: temper- 
ature $T^{*}=T k / \varepsilon$, pressure $p^{*}=p \sigma^{3} / \varepsilon$, density $\rho^{*}=\rho \sigma^{3}$, configurational energy $u^{*}=u / \varepsilon$, configurational enthalpy $h^{*}=h / \varepsilon$, isothermal compressibility $\beta_{T}^{*}=\beta_{T} \varepsilon / \sigma^{3}$, and residual chemical potential as given by equation (4).

For a test of the Grand Equilibrium method on the pure Lennard-Jones fluid, no liquid simulations have been performed in the present work. As for the liquid phase simulations, only standard methods are needed, i.e. NpT ensemble and Widom's test particle insertion, so results from the literature [32] which are known to be accurate were used: Table 1 comprises this data.

Vapour simulations following the Grand Equilibrium method have been performed based on these results. The technical details can be found in the appendix. The vapour-liquid equilibrium results obtained with the Grand Equilibrium method are given in table 2. That table also contains the widely accepted results of Lotfi et al. [32], which were obtained using the NpT plus test particle method [10]. The statistical uncertainties of the present vapour-liquid equilibrium results on the dew line were determined by block averaging [35] of the vapour simulations. In this way, the influence of the uncertainties of the chemical potentials and the partial molar volumes from the liquid simulation is neglected. It should be emphasized that this leads to an underestimation. For a repeatability study, see section 3.2. The statistical uncertainties of the vapour-liquid equilibrium results on the bubble line were determined by block averaging [35] of the liquid simulation and the utilisation of the error propagation law.

It can be seen from table 2 that all data points for vapour pressure, bubble density, and dew density from the present work and those obtained by Lotfi et al. [32] agree within their very small statistical uncertainties. The uncertainties of the Grand Equilibrium method are lower throughout than those of the NpT plus test particle method. In our opinion, this is due to the above mentioned neglecting of the uncertainties of the chemical potentials 
and the partial molar volumes from the liquid simulation, since this is an important source of error and the quality of the results of both methods should be similar.

\subsection{Binary Lennard-Jones mixture}

The Grand Equilibrium method was also tested on a binary Lennard-Jones model mixture, of which the vapour-liquid equilibrium is well known. The parameters of the investigated symmetrical binary model mixture $\mathrm{A}+\mathrm{B}$ are $\sigma_{A}=\sigma_{B}, \varepsilon_{A}=\varepsilon_{B}$, and $\xi_{A B}=0.75$, cf. equations (13)-(15). The thermodynamic quantities are reduced in the same way as for the pure Lennard-Jones fluid, using the parameters of the A-A interaction, $\sigma_{A}$ and $\varepsilon_{A}$. For comparison to the data obtained in the present work, results of the following authors are used: Panagiotopoulos et al. [13] and Tsangaris et al. [36] who applied the Gibbs Ensemble Monte Carlo method (GEMC) as well as Boda et al. [9] who applied the Grand Canonical Monte Carlo method (GCMC).

Binary vapour-liquid equilibrium data is calculated for the two temperatures $T^{*}=1$ and $T^{*}=1.15$. Table 3 comprises the simulation results for the liquid phase. It should be pointed out that all liquid simulations on one isotherm have been performed at the same arbitrarily chosen pressure $p^{l *}$. On the basis of these results, the vapour-liquid equilibria were determined by pseudo- $\mu \mathrm{VT}$ simulations and are given in table 4 . The technical details of the simulations can be found in the appendix. The statistical uncertainties indicated in table 4 were estimated with the same procedure as described above for the pure fluid. In order to investigate the statistical uncertainties of the Grand Equilibrium method appropriately, the vapour-liquid equilibrium for $T^{*}=1.15$ and $x_{A}=0.05$ have been calculated independently four times, cf. table 5. A better estimation of the uncertainties of the vapour-liquid equilibrium can be made by comparing these results. The uncertainties 
are for vapour composition $\delta y_{A}=0.002$, vapour pressure $\delta p_{\sigma}^{*}=0.001$, bubble density $\delta \rho^{\prime *}=0.005$, dew density $\delta \rho^{\prime \prime *}=0.002$, bubble enthalpy $\delta h^{\prime *}=0.03$, and dew enthalpy $\delta h^{\prime \prime *}=0.02$. These values are higher throughout than those obtained by the statistical analysis of the vapour runs, which is easily explained by neglecting of the uncertainties of the chemical potentials and the partial molar volumes from the liquid simulation on the vapour-liquid equilibrium. They are still considerably lower than those reported by Panagiotopoulos et al. [13] for their method.

Different comparisons between the results of the present work obtained using the Grand Equilibrium method and GEMC results of Panagiotopoulos et al. [13] and Tsangaris et al. [36] as well as GCMC results by Boda et al. [9] are given in figures 2-4. From the pressure-composition vapour-liquid equilibrium diagram in figure 2 , it can be seen that the present data shows very little scatter, in the range of less than 0.01 in composition, and that it corresponds very well to the vapour-liquid equilibrium of the pure fluid B at $x_{A}=y_{A}=0$. The agreement with the GEMC results [13,36] is excellent, as almost all vapour-liquid equilibrium points agree with the GEMC results [13,36] within the statistical uncertainties. The agreement with the GCMC results [9] at the lower temperature is good, especially on the dew line. But at the higher temperature considerable deviations occur. For a given pressure, the GCMC [9] compositions on both the bubble and dew line seem to be too low by $0.02-0.03$.

In figure 3 a pressure-density vapour-liquid equilibrium diagram is given. Again, the present data shows very little scatter and is consistent to the results for pure fluid B, for which the saturated densities can be found as the lowest point of each isothermal branch. The agreement to the GEMC results [13,36] is excellent, as all points are within the statistical uncertainties of the GEMC results [13,36]. The dew densities of the GCMC method [9] agree very well with the other data, but the bubble densities of [9] especially 
at the higher temperature are somewhat too high.

Figure 4 shows a pressure-energy density vapour-liquid equilibrium diagram, where the energy density is defined by the product of the configurational energy and the density. Due to the lack of appropriate literature data for $T^{*}=1.15$, only the isotherm $T^{*}=1$ is shown. Once more, it is found that the energy density obtained with the Grand Equilibrium method shows very little scatter and corresponds very well to the limiting pure fluid B, which is included as the lowest point of the present data. The agreement with GEMC [13,36] is again excellent. Consistent with the densities, the GCMC method [9] agrees well on the dew line, whereas on the bubble line, particularly for higher pressures, deviations are observed.

\subsection{Ternary Lennard-Jones mixture}

For a further test, the Grand Equilibrium method was applied to a ternary Lennard-Jones model mixture $\mathrm{A}+\mathrm{B}+\mathrm{C}$, for which vapour-liquid equilibrium results from Tsang et al. 37] using the GEMC method, were available.

That ternary mixture is defined on the basis of equations (13) $-(15)$ by $\sigma_{A}=\sigma_{B}=\sigma_{C}$, $\varepsilon_{B}=0.75 \varepsilon_{A}, \varepsilon_{C}=0.15 \varepsilon_{A}$, and $\xi_{A B}=\xi_{A C}=\xi_{B C}=1$. All thermodynamic quantities are reduced in the usual way, using the parameters of the $\mathrm{A}-\mathrm{A}$ interaction, $\sigma_{A}$ and $\varepsilon_{A}$.

In the first step, liquid simulations for different compositions have been performed at $T^{*}=1$ and $p^{*}=0.2$. The full set of these results is given in table 6 . The vapour-liquid equilibrium data is calculated by pseudo- $\mu \mathrm{VT}$ simulations in the vapour phase as described above, cf. table 7 . The technical details of the simulations are given in the appendix. The lower bounds of the statistical uncertainties of the vapour-liquid equilibrium, as given in table 7 , were estimated by the same procedure as described above for the pure fluid. It 
should be mentioned, that for each vapour-liquid equilibrium point only one simulation in the liquid phase and one simulation in the vapour phase is neccessary, regardless of the number of involved components.

Figure 5 shows a ternary plot of the vapour-liquid equilibrium at $T^{*}=1$ and $p^{*}=0.2$. The results from the present work are compared to the GEMC results of Tsang et al. [37. It can be seen that both data sets are in excellent agreement. 


\section{Conclusion}

A new Grand Equilibrium method for the calculation of vapour-liquid equilibria is presented. It is aimed at mixtures, but can be applied to pure fluids straightforwardly. The independent variables are temperature and liquid composition which is favourable for bubble point calculations.

Two subsequent independent simulation runs have to be performed, first in the liquid, then in the vapour, where standard simulation techniques are utilized. The method requires no prior knowledge of the vapour-lquid equilibrium, is easy to use, and performs reliably.

The Grand Equilibrium method has been applied successfully to the pure Lennard-Jones fluid, a binary, and a ternary mixture. Comparisons to results from other methods show that it yields reliable results with very little scatter. 


\section{Appendix}

For the liquid phase, NpT molecular dynamics simulations were performed, using the algorithm of Andersen [38] and applying periodic boundary conditions as well as the minimum image convention. A cut-off radius of $4 \sigma_{A}$ was introduced, considering the long range corrections. The equations of motion for 500 particles were solved with a fifth-order predictor-corrector method using a time step of 0.005 in usual units. Starting from a f.c.c. lattice, the equilibration period was 5000 time steps, whereof the first 1000 were carried out in the NVT ensemble. The length of the production run was 100000 time steps. In order to calculate the chemical potentials and the partial molar volumes, Widom's test particle method 22] was utilised, by inserting 1000 test particles after each time step. The statistical uncertainties of the simulation results were determined according to the method of Fincham [35].

For the vapour phase, pseudo- $\mu \mathrm{VT}$ Monte Carlo simulations were performed, applying periodic boundary conditions and the minimum image convention. The cut-off radius was again $4 \sigma_{A}$ and the long range corrections were considered. The maximum displacement was set to $5 \%$ of the simulation box length, which was chosen to yield on average 300 to 400 particles in the volume. After 1000 initial NVT loops starting from a f.c.c. lattice, 9000 equilibration loops in the pseudo- $\mu \mathrm{VT}$ ensemble were performed. One loop is defined here to be a number of attempts to displace particles equal to the actual number of particles plus three insertion and three deletion attempts. The length of the production run was 100000 loops. 


\section{Acknowledgements}

The authors thank Mr. Jürgen Stoll for fruitful discussions. We gratefully acknowledge financial support by Deutsche Forschungsgemeinschaft, Sonderforschungsbereich 412, University of Stuttgart. 


\section{References}

[1] Graham, I. S., and Valleau, J. P., 1990, J. Chem. Phys., 96, 193.

[2] Valleau, J. P., 1991, J. Comput. Phys., 94, 7894.

[3] McDonald, I. R., and Singer, K., 1967, Discuss. Faraday Soc., 43, 40.

[4] Potoff, J. J., and Panagiotopoulos, A. Z., 1998, J. Chem. Phys., 109, 10914.

[5] Kofke, D. A., 1993, Molec. Phys., 78, 1331.

[6] Kofke, D. A., 1993, J. Chem. Phys., 98, 4149.

[7] Boda, D., Liszi, J., and Szalai, I., 1995, Chem. Phys. Lett., 235, 140.

[8] Boda, D, Winkelmann, J., Liszi, J., and Szalai. I., 1996, Molec. Phys., 87, 601.

[9] Boda, D., Kristóf, T., Liszi, J., and Szalai, I., 2001, Molec. Phys., 99, 2011.

[10] Möller, D., and Fischer, J., 1990, Molec. Phys., 69, 463.

[11] Vrabec, J., and Fischer, J., 1995, Molec. Phys., 85, 781.

[12] Panagiotopoulos, A. Z., 1987, Mol. Phys., 61, 813.

[13] Panagiotopoulos, A. Z., Quirke, N., Stapleton, M., and Tildesley, D. J., 1988, Mol. Phys., 63, 527.

[14] Panagiotopoulos, A. Z., 1992, Fluid Phase Equilibria, 76, 97.

[15] Baus, M., Rull., L. F., and Ryckaert, J. P., 1995, Observation, Prediction and Simulation of Phase Transitions in Complex Fluids, (Dordrecht Kluver).

[16] Vrabec, J., and Fischer, J., 1996, AIChE J., 43, 212.

[17] Ungerer, P., Boutin, A., and Fuchs, A. H., 1999, Molec. Phys., 97, 523.

[18] Ungerer, P., Boutin, A., and Fuchs, A. H., 2001, Molec.Phys., 99, 1423.

[19] Escobedo, F. A., 1998, J. Chem. Phys., 108, 8761.

[20] Escobedo, F. A., 1999, J. Chem. Phys., 110, 11999.

[21] Hill, T. L., 1956, Statistical Mechanics (New York: McGraw-Hill Book Company).

[22] Widom, B., 1963, J. Chem. Phys., 39, 2808.

[23] Heyes, D. M., 1992, Mol. Simul., 8, 227. 
[24] Shevkunov, S. V., Martinovski, A. A., and Vorontsov-Velyaminov, P. N., 1988, High Temp. Phys. (USSR), 26, 246.

[25] Lyubartsev, A. P., Martinovski, A. A., Shevkunov, S. V., and Vorontsov-Velyaminov, P. N., 1992, J. Chem. Phys., 96, 1776.

[26] Nezbeda, I., and Kolafa, J., 1991, Mol. Simul., 5, 391.

[27] Allen, M. P., and Tildesley, D. J., 1987, Computer simulations of liquids (Oxford: Clarendon Press).

[28] Frenkel, D., and Smit, B., 1996, Understanding Molecular Simulation (San Diego: Academic Press).

[29] Sadus, R. J., 1999, Molecular Simulation of Fluid: Theory, Algorithms, and Objectorientation (Amsterdam: Elsevier).

[30] Kristof, T., Liszi, J., and Boda, D., 2002, Molec. Phys., submitted.

[31] Stoll, J., Vrabec, J., and Hasse, H., 2002, AIChE J., submitted.

[32] Lotfi, A., Vrabec, J., and Fischer, J., 1992, Molec. Phys., 76, 1319.

[33] Vrabec, J., Lotfi, A., and Fischer, J., 1993, Fluid Phase Equilibria, 89, 383.

[34] Okumura, H., and Yonezawa, F., 2000, J. Chem. Phys. 113, 9162.

[35] Fincham, D., Quirke, N., and Tildesley, D. J., 1986, J. Chem. Phys., 84, 4535.

[36] Tsangaris, D. M., and McMahon, P. D., 1992, Mol. Simul., 9, 223.

[37] Tsang, P. C., White, O. N., Perigard, B. Y., Vega, L. F., and Panagiotopoulos, A. Z., 1995, Fluid Phase Equilibria, 107, 31.

[38] Andersen, H. C., 1980, J Chem. Phys., 72, 2384. 
Table 1

Thermodynamic properties in the liquid phase of the pure Lennard-Jones fluid taken from Lotfi et al. 32. The numbers in paranthesis denote the uncertainty in the last digit.

\begin{tabular}{cc|ccccc}
\hline$T^{*}$ & $p^{*}$ & \multicolumn{2}{|c}{$\tilde{\mu}$} & $\rho^{*}$ & \multicolumn{2}{c}{$\beta_{T}^{*}$} \\
\hline 0.75 & 0.00 & -5.69 & $(3)$ & $0.8214(4)$ & $0.091(3)$ \\
1.00 & 0.03 & $-3.823(7)$ & $0.7018(4)$ & 0.28 & $(2)$ \\
1.15 & 0.06 & $-3.200(4)$ & $0.6056(6)$ & 0.6 & $(1)$ \\
1.25 & 0.10 & $-2.882(4)$ & 0.516 & $(2)$ & 2.3 & $(5)$ \\
\hline
\end{tabular}


Table 2

Comparison of vapour-liquid equilibria results of the pure Lennard-Jones fluid from the present work (Grand Equilibrium method) and from Lotfi et al. [32] (NpT plus test particle method). The numbers in paranthesis denote the uncertainty in the last digit.

\begin{tabular}{|c|c|c|c|c|}
\hline$T^{*}$ & $p_{\sigma}^{*}$ & $\rho^{\prime *}$ & $\rho^{\prime \prime *}$ & \\
\hline \multirow[t]{2}{*}{0.75} & $0.00261(1)$ & $0.8216(4)$ & $0.00359(1)$ & present work \\
\hline & $0.00264(7)$ & $0.8216(4)$ & $0.0036 \quad(1)$ & 32 \\
\hline \multirow[t]{2}{*}{1.00} & $0.02500(4)$ & $0.7008(4)$ & $0.0296 \quad(1)$ & present work \\
\hline & $0.0250 \quad(2)$ & $0.7008(4)$ & $0.0296 \quad(3)$ & 32 \\
\hline \multirow[t]{2}{*}{1.15} & $0.0597 \quad(2)$ & $0.6054(6)$ & $0.0728 \quad(4)$ & present work \\
\hline & $0.0597 \quad(4)$ & $0.6055(7)$ & $0.0727 \quad(8)$ & 32 \\
\hline \multirow[t]{2}{*}{1.25} & $0.0971 \quad(5)$ & $0.512 \quad(2)$ & 0.136 & present work \\
\hline & 0.097 & $0.513 \quad(3)$ & 0.134 & 32 \\
\hline
\end{tabular}


Table 3

Thermodynamic properties in the liquid phase of the binary Lennard-Jones mixture $\sigma_{A}=\sigma_{B}$, $\varepsilon_{A}=\varepsilon_{B}, \xi_{A B}=0.75$. The numbers in paranthesis denote the uncertainty in the last digit.

\begin{tabular}{|c|c|c|c|c|c|c|c|c|}
\hline$x_{A}$ & $\tilde{\mu}_{A}$ & $\tilde{\mu}_{B}$ & $v_{A}^{*}$ & $v_{B}^{*}$ & $\rho^{*}$ & $h^{*}$ & $\beta_{T}^{*}$ & $\left(\frac{\partial h^{*}}{\partial p^{*}}\right)_{T, \mathbf{x}}$ \\
\hline \multicolumn{9}{|c|}{$T^{*}=1, p^{l *}=0.04$} \\
\hline 0.05 & $-4.73 \quad(1)$ & $-3.87 \quad(1)$ & $2.0(1)$ & $1.3(1)$ & $0.6892(6)$ & $-5.637(7)$ & $0.29(1)$ & $0.13(6)$ \\
\hline 0.10 & $-4.302(8)$ & $-3.908(8)$ & $1.8(1)$ & $1.2(1)$ & $0.6744(5)$ & $-5.428(6)$ & $0.31(2)$ & $0.14(7)$ \\
\hline 0.15 & $-4.140(8)$ & $-3.931(8)$ & $1.9(1)$ & $1.3(1)$ & $0.6618(6)$ & $-5.262(6)$ & $0.37(2)$ & $-0.02(7)$ \\
\hline 0.20 & $-4.042(8)$ & $-3.940(7)$ & $2.1(2)$ & $1.5(1)$ & $0.6506(7)$ & $-5.11 \quad(1)$ & $0.47(2)$ & $-0.3 \quad(1)$ \\
\hline \multicolumn{9}{|c|}{$T^{*}=1.15, p^{l *}=0.08$} \\
\hline 0.05 & $-4.641(8)$ & $-3.226(6)$ & $3.0(2)$ & $1.6(1)$ & $0.587 \quad(1)$ & $-4.96 \quad(3)$ & $0.84(4)$ & $-1.5 \quad(2)$ \\
\hline 0.10 & $-4.181(5)$ & $-3.270(4)$ & $2.7(2)$ & $1.3(1)$ & $0.559 \quad(1)$ & $-4.68 \quad(3)$ & $1.03(6)$ & $-1.9 \quad(2)$ \\
\hline 0.15 & $-3.952(6)$ & $-3.294(4)$ & $3.8(3)$ & $1.6(1)$ & $0.534 \quad(2)$ & $-4.45 \quad(5)$ & $2.0 \quad(2)$ & $-4.6 \quad(5)$ \\
\hline 0.20 & $-3.833(6)$ & $-3.306(3)$ & $4.4(4)$ & $1.3(2)$ & $0.497 \quad(3)$ & $-4.17 \quad(7)$ & $3.9 \quad(4)$ & (1) \\
\hline
\end{tabular}


Table 4

Vapour-liquid equilibria of the binary Lennard-Jones mixture $\sigma_{A}=\sigma_{B}, \varepsilon_{A}=\varepsilon_{B}, \xi_{A B}=0.75$.

The numbers in paranthesis denote the uncertainty in the last digit.

\begin{tabular}{|c|c|c|c|c|c|c|}
\hline$x_{A}$ & $y_{A}$ & $p_{\sigma}^{*}$ & $\rho^{\prime *}$ & $\rho^{\prime \prime *}$ & $h^{\prime *}$ & $h^{\prime \prime *}$ \\
\hline \multicolumn{7}{|c|}{$T^{*}=1$} \\
\hline 0.05 & $0.275(2)$ & $0.0346(1)$ & $0.6881(6)$ & $0.0424(1)$ & $-5.638(7)$ & $-0.524(2)$ \\
\hline 0.10 & $0.390(2)$ & $0.0403(1)$ & $0.6745(5)$ & $0.0513(2)$ & $-5.428(6)$ & $-0.611(2)$ \\
\hline 0.15 & $0.438(2)$ & $0.0436(1)$ & $0.6627(6)$ & $0.0568(2)$ & $-5.262(6)$ & $-0.669(3)$ \\
\hline 0.20 & $0.472(2)$ & $0.0467(2)$ & $0.6526(8)$ & $0.0627(3)$ & $-5.11 \quad(1)$ & -0.735 \\
\hline \multicolumn{7}{|c|}{$T^{*}=1.15$} \\
\hline 0.05 & $0.158(1)$ & $0.0722(2)$ & $0.584 \quad(1)$ & $0.0911(4)$ & $-4.95 \quad(3)$ & $-1.064(5)$ \\
\hline 0.10 & $0.248(2)$ & $0.0806(3)$ & $0.559 \quad(1)$ & $0.1051(6)$ & $-4.68 \quad(3)$ & $-1.158(7)$ \\
\hline 0.15 & $0.308(2)$ & 0.0890 & $0.543 \quad(2)$ & $0.124 \quad(1)$ & $-4.49 \quad(5)$ & $-1.33 \quad(1)$ \\
\hline 0.20 & $0.341(2)$ & 0.0958 & $0.528 \quad(5)$ & $0.144 \quad(1)$ & $-4.32 \quad(7)$ & $-1.50 \quad(1)$ \\
\hline
\end{tabular}


Table 5

Vapour-liquid equilibria of the binary Lennard-Jones mixture $\sigma_{A}=\sigma_{B}, \varepsilon_{A}=\varepsilon_{B}, \xi_{A B}=0.75$ for the state point $T^{*}=1.15$ and $x_{A}=0.05$ from independent simulation runs. The numbers in paranthesis denote the uncertainty in the last digit.

\begin{tabular}{cccccc}
\hline$y_{A}$ & $p_{\sigma}^{*}$ & $\rho^{\prime *}$ & $\rho^{\prime *}$ & $h^{\prime *}$ & $h^{\prime \prime *}$ \\
\hline $0.160(1)$ & $0.0732(3)$ & $0.588(1)$ & $0.0931(5)$ & $-4.97(3)$ & $-1.081(7)$ \\
$0.159(1)$ & $0.0727(2)$ & $0.584(1)$ & $0.0921(5)$ & $-4.96(3)$ & $-1.071(7)$ \\
$0.159(2)$ & $0.0720(3)$ & $0.583(1)$ & $0.0910(6)$ & $-4.94(3)$ & $-1.060(8)$ \\
$0.158(1)$ & $0.0722(2)$ & $0.584(1)$ & $0.0911(4)$ & $-4.95(3)$ & $-1.064(5)$ \\
\hline
\end{tabular}


Table 6. Thermodynamic properties in the liquid phase of the ternary Lennard-Jones mixture $\sigma_{A}=\sigma_{B}=\sigma_{C}, \varepsilon_{B}=0.75 \varepsilon_{A}$, $\varepsilon_{C}=0.15 \varepsilon_{A}, \xi_{A B}=\xi_{A C}=\xi_{B C}=1$ at the temperature $T^{*}=1$ and the pressure $p^{l *}=0.2$. The numbers in paranthesis denote the uncertainty in the last digit.

\begin{tabular}{ccc|cccccccccccccc} 
& \multicolumn{1}{c|}{$x_{A}$} & $x_{B}$ & \multicolumn{1}{c}{$\tilde{\mu}_{A}$} & $\tilde{\mu}_{B}$ & $\tilde{\mu}_{C}$ & $v_{A}^{*}$ & $v_{B}^{*}$ & $v_{C}^{*}$ & $\rho^{*}$ & $h^{*}$ & $\beta_{T}^{*}$ & $\left(\frac{\partial h^{*}}{\partial p^{*}}\right)_{T, \mathbf{x}}$ \\
\hline & 0.972 & 0 & -3.61 & $(1)$ & & -1.69 & $(1)$ & $1.2(1)$ & & $2.3(1)$ & $0.7114(4)$ & $-5.50(2)$ & $0.202(7)$ & $0.43(4)$ \\
& 0.724 & 0.232 & $-3.890(8)$ & $-3.869(8)$ & -1.79 & $(1)$ & $1.4(1)$ & $1.8(1)$ & $3.1(2)$ & $0.6663(6)$ & $-4.79(3)$ & 0.35 & $(2)$ & $0.05(7)$ \\
& 0.432 & 0.492 & $-4.282(5)$ & $-3.183(5)$ & $-1.954(9)$ & $1.1(1)$ & $1.8(1)$ & $4.0(2)$ & $0.5876(8)$ & $-3.83(6)$ & 0.74 & $(4)$ & -0.7 & $(1)$ \\
& 0.312 & 0.590 & $-4.505(3)$ & $-3.007(4)$ & $-2.085(8)$ & $0.6(1)$ & $1.6(1)$ & $5.1(3)$ & 0.533 & $(7)$ & $-3.33(4)$ & 1.31 & $(7)$ & -1.8 & $(2)$ \\
\hline
\end{tabular}


Table 7

Vapour-liquid equilibria of the ternary Lennard-Jones mixture $\sigma_{A}=\sigma_{B}=\sigma_{C}, \varepsilon_{B}=0.75 \varepsilon_{A}$, $\varepsilon_{C}=0.15 \varepsilon_{A}, \xi_{A B}=\xi_{A C}=\xi_{B C}=1$ at the temperature $T^{*}=1$. The numbers in paranthesis denote the uncertainty in the last digit.

\begin{tabular}{|c|c|c|c|c|c|c|c|c|}
\hline$x_{A}$ & $x_{B}$ & $y_{A}$ & $y_{B}$ & $p_{\sigma}^{*}$ & $\rho^{\prime *}$ & $\rho^{\prime \prime *}$ & $h^{\prime *}$ & $h^{\prime \prime *}$ \\
\hline 0.972 & 0 & $0.245(3)$ & 0 & $0.1978(8)$ & $0.7111(4)$ & $0.1893(8)$ & $-5.50(2)$ & $-0.297(7)$ \\
\hline 0.724 & 0.232 & $0.205(3)$ & $0.168(2)$ & $0.207 \quad(1)$ & $0.6680(7)$ & $0.211 \quad(1)$ & $-4.79(3)$ & $-0.510(8)$ \\
\hline 0.432 & 0.492 & $0.169(4)$ & $0.369(3)$ & $0.212 \quad(1)$ & $0.593 \quad(1)$ & $0.248 \quad(2)$ & $-3.84(6)$ & $-0.76 \quad(1)$ \\
\hline 0.312 & 0.590 & $0.147(5)$ & $0.457(3)$ & $0.200 \quad(1)$ & $0.532 \quad(1)$ & $0.250 \quad(2)$ & $-3.33(9)$ & $-1.05 \quad(2)$ \\
\hline
\end{tabular}




\section{List of Figures}

1 Pressure, density, and vapour composition over Monte-Carlo loops during the equilibration period.

2 Vapour-liquid equilibrium diagram of the binary Lennard-Jones mixture $\sigma_{A}=\sigma_{B}, \varepsilon_{A}=\varepsilon_{B}, \xi_{A B}=0.75$ at $T^{*}=1$ and 1.15. $\bullet$ Grand Equilibrium method (this work), $\boldsymbol{\Delta}$ GEMC results (Tsangaris et al. [36]), $\mathbf{\nabla}$ GEMC results (Panagiotopoulos et al. [13]), - GCMC results (Boda et al. [9]).

3 Vapour-liquid equilibrium diagram of the binary Lennard-Jones mixture $\sigma_{A}=\sigma_{B}, \varepsilon_{A}=\varepsilon_{B}, \xi_{A B}=0.75$ at $T^{*}=1$ and 1.15. Left: dew densities. Right: bubble densities. • Grand Equilibrium method (this work), GEMC results (Tsangaris et al. [36]), $\mathbf{\nabla}$ GEMC results (Panagiotopoulos et al. [13]), — GCMC results (Boda et al. [9]).

4 Vapour-liquid equilibrium diagram of the binary Lennard-Jones mixture $\sigma_{A}=\sigma_{B}, \varepsilon_{A}=\varepsilon_{B}, \xi_{A B}=0.75$ at $T^{*}=1$. Left: bubble energy densities. Right: dew energy densities. • Grand Equilibrium method (this work), GEMC results (Tsangaris et al. [36]), — GCMC results (Boda et al. [9]).

5 Vapour-liquid equilibrium of the ternary Lennard-Jones mixture $\sigma_{A}=\sigma_{B}=\sigma_{C}, \varepsilon_{B}=0.75 \varepsilon_{A}, \varepsilon_{C}=0.15 \varepsilon_{A}, \xi_{A B}=\xi_{A C}=\xi_{B C}=1$ at $T^{*}=1$ and $p^{*}=0.2$. Grand Equilibrium method (this work), $\Delta$ GEMC results (Tsang et al. [37]), — conode. 
Fig. 1.

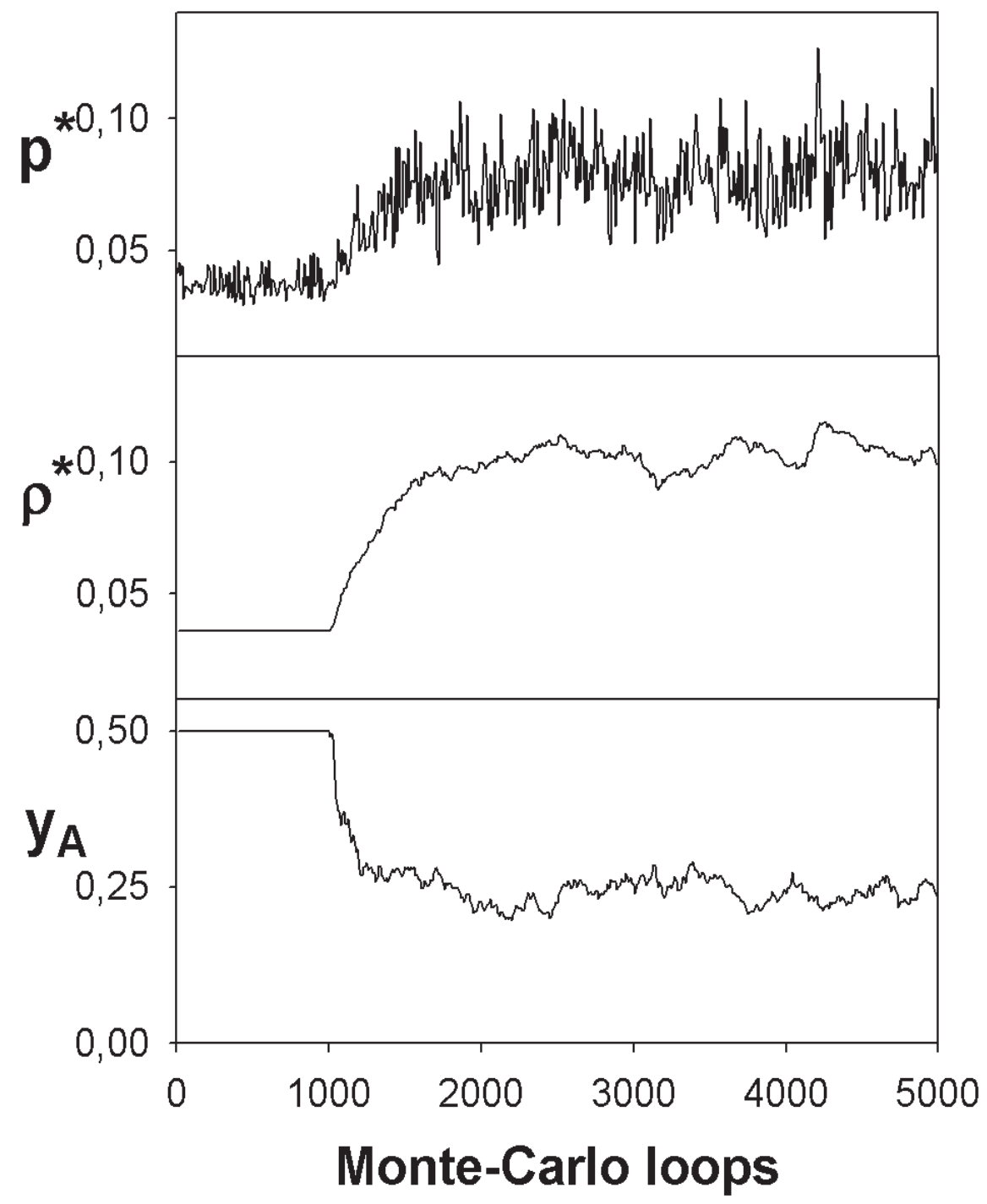


Fig. 2.

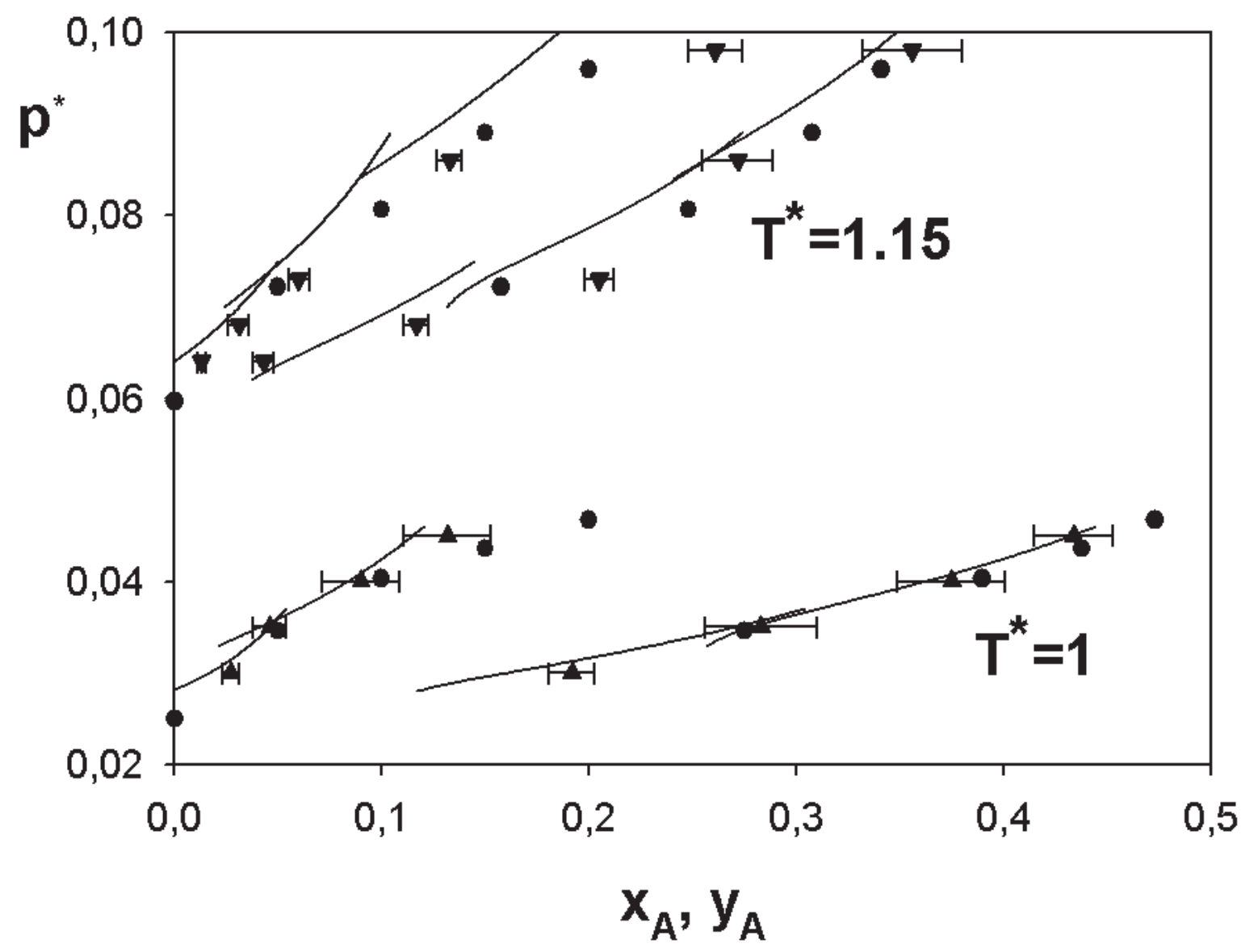


Fig. 3.

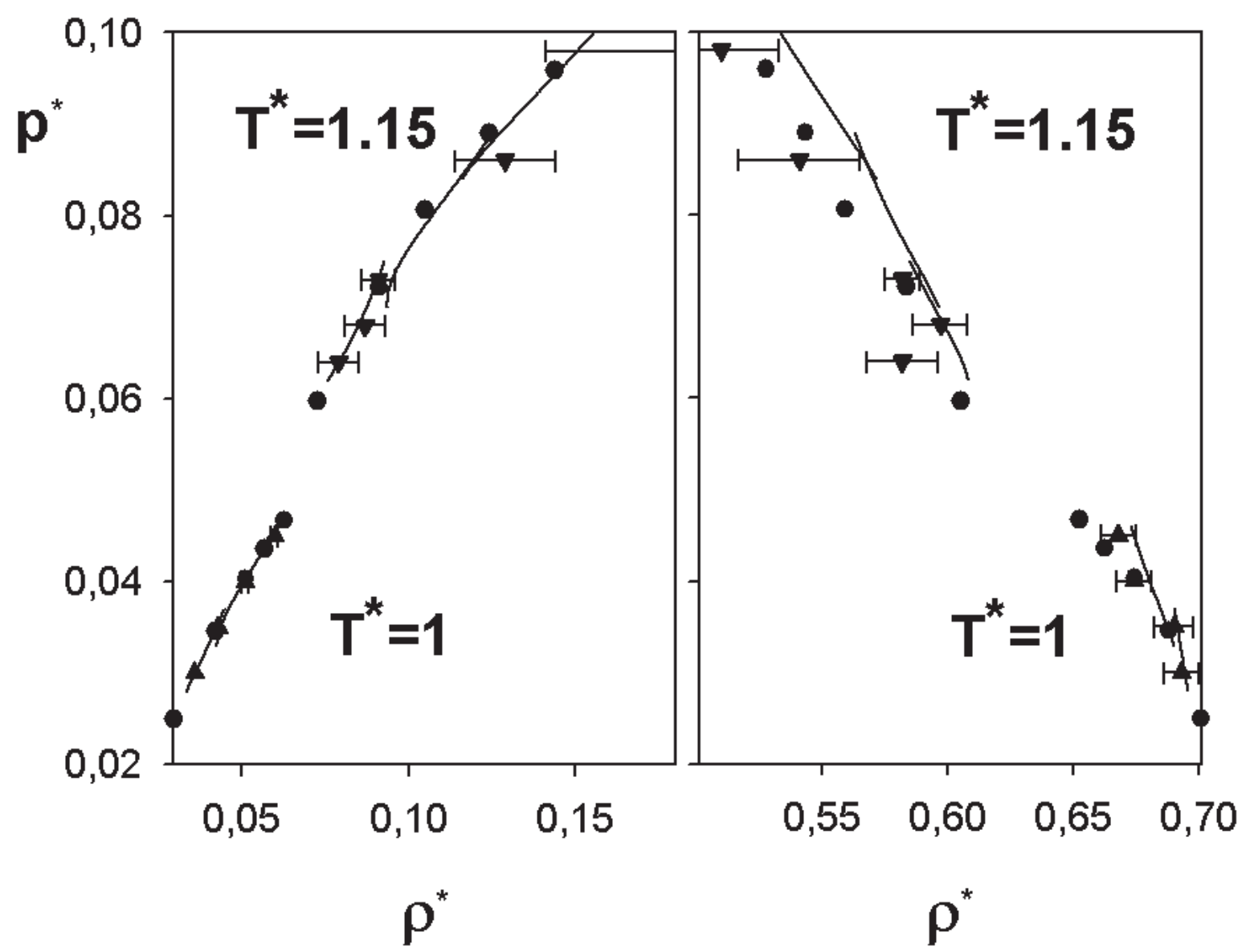


Fig. 4.

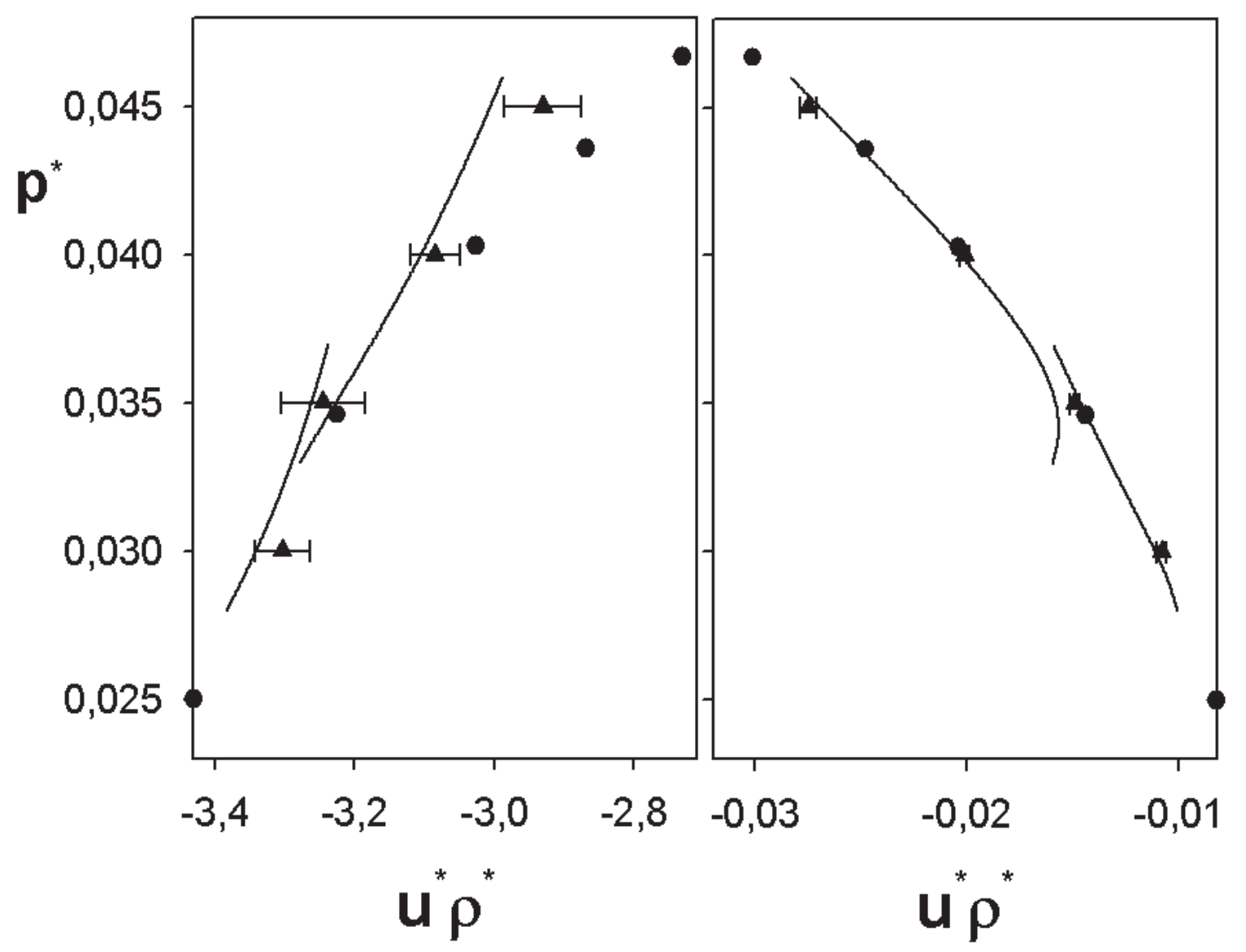


Fig. 5.
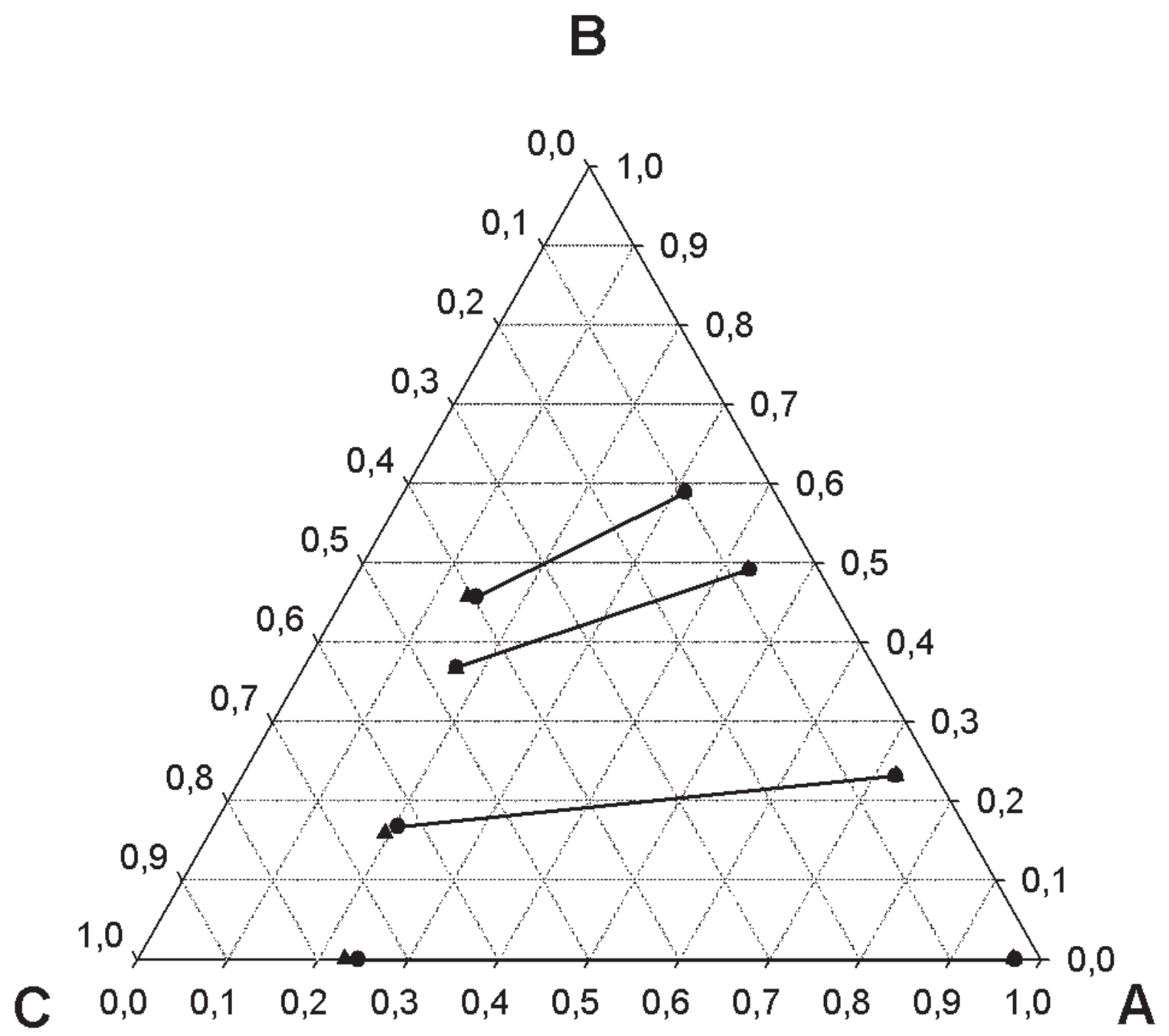